\section{CIENCIA Y ECONOMÍA}

\author{
Andrés Barge-Gil y Aurelia Modrego Rico \\ Laboratorio de Análisis y Evaluación del Cambio Técnico \\ Universidad Carlos III de Madrid
}

\title{
SCIENCE AND ECONOMY
}

\begin{abstract}
The relationship between science and economy is a tren$d y$ issue, both in the scientific field as in the entrepreneurial and political arena. The main purpose of this work is to show in a synthetic way the state of the art of this matter. To this aim, we put forward the analysis of science developed by economists, focusing in the controversies between the neoclassical and the evolutionary perspective. We also review the empirical evidence offered by different works and sum up the main arguments of the present debate about how science should be organized. Main conclusions show that science and economy are closely related and that their links are complex and dynamic, so that flexible approaches are required in the management and evaluation of the relationship.
\end{abstract}

KEY WORDS: Science, economy, innovation, impact, evolutionary.

\section{INTRODUCCIÓN}

El reconocimiento de la relación entre ciencia y economía por parte de un amplio sector de la sociedad no se produjo hasta la Segunda Guerra Mundial. El prestigio alcanzado por la ciencia como consecuencia del decisivo papel desempeñado en la contienda bélica, tanto en sus aspectos destructivos (la bomba atómica o los misiles de largo alcance) como en su ayuda para salvar vidas (la penicilina), supuso una justificación para la implementación de apoyo político a gran escala para las actividades de investigación científica. El informe de Vannevar Bush (1945): Science: The Endless Frontier (Ciencia: la frontera sin límite) sentó las bases del modelo científico dominante en los siguientes treinta años, que se caracterizó por lo que algunos autores han denominado la "República de la ciencia" (Polanyi, 1962), reflejo de la autonomía que la sociedad otorgaba a los científicos a la hora de determinar sus agendas de investigación, así como sus sistemas de incentivos y de evaluación.
RESUMEN: La relación entre ciencia y economía es un tema de gran actualidad, atrae la atención tanto en el mundo científico como en el empresarial y el político. El principal objetivo de este trabajo consiste en mostrar de forma sintética el estado del arte sobre esta cuestión. Para ello, se presenta el análisis de la ciencia realizado por los economistas, prestando especial atención a las controversias existentes entre la perspectiva neoclásica y evolucionista, se repasa la evidencia empírica existente a partir de trabajos de muy distinta naturaleza y se resumen las líneas principales del debate actual acerca el modelo de organización de la ciencia. Las principales conclusiones del estudio muestran que ciencia y economía se hallan intimamente relacionadas y que sus vínculos son complejos y dinámicos, por lo que se requieren planteamientos flexibles en la gestión y evaluación de dicha relación.

PALABRAS CLAVE: Ciencia, economía, innovación, impacto, evolucionista.

A partir de la década de los ochenta algunas circunstancias, como el incremento de la competencia económica internacional, las restricciones del gasto público y la mayor importancia de las capacidades y habilidades científicotecnológicas como recursos estratégicos de las empresas (Martin, 2003), provocaron un cambio en la mentalidad social en relación con la ciencia. Esto se tradujo en una reducción importante del apoyo de algunos gobiernos a la ciencia, especialmente en los recursos destinados a las instituciones académicas para el desarrollo de la investigación fundamental, con la expectativa de que este reajuste fuera compensado por la industria. Las universidades fueron animadas a buscar apoyo en el sector empresarial cualquiera que fuera la forma de conseguirlo. El resultado fue que en muchos países el presupuesto de I+D en el sector académico como porcentaje del producto nacional bruto en I+D se redujo, en algunos casos de forma significativa.

Este cambio sustancial ha sido descrito como el resultado de la evolución de la demanda social de producción de conocimiento desde un Modo 1 hacia un Modo 2 (Gibbons 
et al., 1994). El Modo 1 se caracteriza por la existencia de una escasa conexión entre las necesidades de la sociedad y las orientaciones de las investigaciones científicas. Por el contrario, el Modo 2 plantea claramente un hecho diferencial respecto del Modo 1 basado en la idea de que la producción de conocimiento se ha de llevar a cabo con la intención de ser aplicado para resolver las necesidades sociales. Este planteamiento ha tenido una gran repercusión en el ámbito universitario, y ha sido interpretado como la incorporación de una tercera misión (además de la investigación básica y la formación) a los objetivos de las universidades (Leyesdorff y Etzkowitz, 1996).

Esta situación no es más que una plasmación de la complejidad que plantea el análisis de la relación entre ciencia y economía, íntimamente ligada a los avatares económicos, sociales y culturales. Varios autores (Rosenberg y Nelson, 1994; Martin, 2003) argumentan que este cambio supone un retorno a la situación de la ciencia en el siglo XIX y a principios del siglo XX. Ésta es la nueva dinámica en la que se halla inmersa la comunidad científica, a la que con una presión creciente se le pide que oriente sus esfuerzos a la resolución de problemas de la sociedad y obtenga una valorización económica de los resultados de sus investigaciones.

Es en este contexto en el que resulta pertinente detenerse a reflexionar acerca de las aproximaciones más relevantes que se han utilizado para abordar el análisis de las características de la relación entre la ciencia y la economía. El presente trabajo pretende desempeñar esta tarea a partir de una revisión detallada de los principales argumentos y resultados ofrecidos por los principales estudios en este ámbito. Por limitaciones de espacio no se aborda con detalle las repercusiones que han tenido los distintos enfoques en los diseños de las políticas públicas. La estructura de la exposición es la siguiente. En el próximo apartado se presentan las distintas formas en que la economía ha conceptualizado y caracterizado la actividad científica. A continuación se realiza una revisión de los diferentes estudios empíricos que se han planteado la medición de la relación entre ciencia y economía. En el cuarto apartado, se presenta el importante debate que se está desarrollando actualmente, entre los partidarios de un modelo de ciencia abierta y aquellos que demandan extender los derechos de propiedad industrial. Por último, se ofrecen las principales conclusiones del trabajo.

\section{La CONCEPTUALIZACIÓN ECONÓMICA DE LA CIENCIA}

Desde el enfoque neoclásico de la economía, se ha considerado que el resultado fundamental de la actividad científica es el conocimiento, un bien que tiene una propiedad inherente a los bienes públicos: la no rivalidad en su uso. Esto supone que, a diferencia de un bien económico normal, muchos agentes pueden hacer uso a la vez del mismo conocimiento (por ejemplo: el teorema de Pitágoras) sin que ninguno de ellos se vea afectado negativamente. A esto hay que añadir otra propiedad relacionada con el funcionamiento del mercado, ya que si éste opera libremente, una vez que el conocimiento se difunde cualquier agente puede utilizarlo, aunque no haya intervenido en su creación.

Esta situación implica un problema adicional, que es el de apropiabilidad. Como consecuencia, los posibles beneficios económicos derivados de la inversión en ciencia no podrán ser completamente apropiados por el inversor, ya que otros agentes podrán utilizar libremente el conocimiento generado para su propio beneficio sin necesidad de haber incurrido en ningún gasto. En estas condiciones en las que el beneficio social de la inversión en ciencia excede al beneficio privado obtenido por el inversor y los incentivos con los que el mercado provee a los agentes para invertir en ciencia son reducidos, la producción científica que se obtiene es inferior a la socialmente óptima. A esto se añaden otras dos características del conocimiento: la elevada incertidumbre a la que se halla sujeto su proceso de producción y la existencia de indivisibilidades en el mismo (Arrow, 1962; Dasgupta, 1987).

A pesar de que estas peculiaridades habian sido reconocidas desde el principio en los trabajos académicos, en la práctica el conocimiento se consideró un "bien público duradero" (Dasgupta and David, 1994, p. 491) y se analizó de la misma forma que otros bienes públicos duraderos como, por ejemplo, las farolas o las leyes. Esta aproximación a la actividad científica es la que la economía ha considerado tradicionalmente, a partir de los estudios seminales de Nelson (1959) y Arrow (1962), y la existencia de "fallos de mercado" ha servido para justificar la intervención pública con la finalidad de subsanar los problemas que el mercado no puede resolver.

Es el propio Nelson (2006), junto a otros autores, quien ha reconocido años después que el rango de instituciones 
implicadas en la producción y difusión del conocimiento científico es muy amplio, e incluye tanto a instituciones que operan en el mercado como a instituciones que no lo hacen. De esta forma, la existencia de esta variedad institucional debe ser considerada como una parte sustancial en el análisis económico de la ciencia; en otro caso, la comprensión de las instituciones ajenas al mercado implicadas en el desarrollo de la ciencia como respuestas a fallos del mercado pueden llevar a una interpretación "asimétrica y distorsionadora" (Nelson, 2006, p. 905) de la realidad.

Algunos de los argumentos anteriores también se hallan recogidos en el trabajo de otros economistas que han desarrollado una "nueva economía de la ciencia" (Dasgupta and David, 1994; Stephan, 1996), que, apoyada en el trabajo de sociólogos y filósofos, incorpora el análisis de las normas y costumbres en las comunidades científicas y de la producción de conocimiento. Entre ellas destacan los sistemas de incentivos propios a las mismas, las peculiaridades de los mercados de trabajo de científicos y la manera más adecuada de financiar y gestionar estas actividades.

Esta nueva economía de la ciencia se puede ubicar en un enfoque alternativo de la economía neoclásica: el enfoque evolucionista. Los intentos de concebir la economía desde una perspectiva de evolución tienen en su origen en los trabajos de Veblen (1898) y de otros institucionalistas americanos, de Marshall (1890) y, fundamentalmente, de Schumpeter $(1912 ; 1943)$ y han adquirido un creciente protagonismo en los últimos años, a partir de la publicación del libro An Evolutionary Theory of Economic Change (Nelson and Winter, 1982).

La economía evolucionista critica los fallos de mercado, ya que considera que la noción de equilibrio implícita en dicha aproximación tiene un marcado carácter estático, por lo que en el caso de la actividad científica y de los procesos de innovación, cuyas características principales son su dinamismo y búsqueda de novedad (Nelson y Soete, 1988), esta visión difícilmente puede considerarse como válida para tratar de una forma global sus peculiaridades.

En el estudio del conocimiento como bien económico, los fallos de mercado están en todas partes, resultan ser la norma, no la excepción, y, por ello, no resultan útiles como únicos instrumentos de análisis ni de justificación de las políticas públicas. En esta línea, Metcalfe $(1995,1997)$ considera fundamental el papel de la incertidumbre y llama la atención sobre el insuficiente tratamiento prestado en la aproximación neoclásica. Es más, la existencia de incertidumbre y asimetrías de información es una condición necesaria para la creación de nuevo conocimiento (Dosi, 1988), por lo que considerarlas un fallo del mercado resulta una contradicción.

En contraposición con el enfoque neoclásico, el evolucionista estudia "trayectorias dinámicas en el tiempo" (Dosi y Nelson, 1998). Entre sus principales caracteristicas (Dosi, 1988) se pueden identificar algunas que resultan muy adecuadas entender el proceso de producción científica: los agentes son heterogéneos y tienen distintas capacidades, el conocimiento tiene un componente tácito que se halla incorporado en las personas $y$, también, en las organizaciones, la situación actual depende del pasado, las interacciones entre agentes son fundamentales para explicar los resultados, las instituciones no económicas son muy relevantes y la economía debería prestar más atención al diálogo con otras disciplinas como la historia, la sociología o la filosofía.

A esto hay que añadir el hecho de que la economía neoclásica ha propiciado el tratamiento del conocimiento científico como algo exógeno, y considera que su influencia en el crecimiento económico tiene lugar de una manera secuencial o lineal: los nuevos descubrimientos científicos llevan a nuevos desarrollos tecnológicos que, al introducirse en el mercado a través de nuevos productos y procesos, conllevan beneficios económicos (Kline y Rosenberg, 1986). Sin embargo, desde el enfoque evolucionista, se considera que este modelo tan simplificado no es representativo de la realidad. Por una parte, no reconoce suficientemente la importancia de las retroalimentaciones en el proceso (desde el mercado o la tecnología hasta la ciencia) ni el aprendizaje que se genera; sin embargo, ambos elementos son de gran relevancia tanto en su contribución al planteamiento de nuevos problemas y retos para la investigación científica, como en su apoyo a los procesos de investigación a partir del suministro de nuevos instrumentos y herramientas más potentes y precisas. Por otra parte, no tiene en cuenta que la mayoría de las innovaciones económicamente importantes no utilizan nuevos descubrimientos científicos de forma inmediata. Por el contrario, se basan generalmente en la base de conocimiento disponible

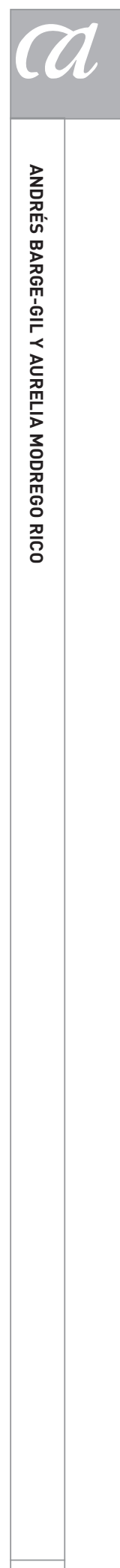

ARBOR CLXXXV 738 julio-agosto [2009] 757-766 ISSN: 0210-1963 
("ciencia vieja"), lo que conlleva realizar un gran número de actividades complejas y costosas (como vigilancia, formación, diseño, etc.) que permiten reconocer y valorar qué tipo de conocimiento está disponible, adaptarlo a los fines específicos que se persiguen, integrarlo con el resto de funciones de la empresa, ponerlo en valor, y conseguir obtener beneficios del mismo (Bender y Laestadius, 2005). Este tipo de actividades son las que se suelen incluir en el concepto de "capacidad de absorción" (Cohen and Levinthal, 1989; 1990; Lane et al., 2006) que establece que el desarrollo de las capacidades internas de las organizaciones es necesario para obtener el máximo provecho del conocimiento existente en otros agentes'.

\section{LA EVIDENCIA EMPÍRICA}

A efectos empíricos, el objetivo es desarrollar métodos para medir la relación entre ciencia y economía. Algunos autores (Salter y Martin, 2001) proponen dividir los estudios empíricos que analizan la importancia de la ciencia en el crecimiento económico en dos grandes grupos de trabajos: aquellos que utilizan una perspectiva econométrica y los que emplean la información obtenida a partir de encuestas o estudios de caso (Salter y Martin, 2001)2.

\subsection{Estudios econométricos}

Dentro de los estudios econométricos se pueden diferenciar tres grandes grupos, en función de la perspectiva adoptada: macroeconómica, mesoeconómica y microeconómica.

Un primer grupo de estudios econométricos está basado en los trabajos de la macroeconomía neoclásica del crecimiento, en los que se utiliza una función de producción agregada para estimar la evolución del producto de una economía en función de la utilización de los factores productivos tradicionales (trabajo y capital). Los primeros modelos trataron el cambio tecnológico como un residuo, es decir, como la parte del crecimiento que no podía ser explicada por los factores productivos clásicos (Solow, 1957). Posteriormente, la denominada "nueva teoría del crecimiento" (Grossman y Helpman, 1989; Romer, 1990) han tratado de estimar la influencia de la tecnología y de la investigación en el crecimiento del producto, instrumentalizándola como un nuevo factor productivo. Tanto la primera como la segunda generación de modelos muestran que dicha influencia es muy relevante. Sin embargo, es preciso reconocer que los supuesto de partida utilizados son muy restrictivos y que los indicadores empleados son poco fiables (Verspagen, 1992.) ya que, por ejemplo, no permiten distinguir entre la influencia de la ciencia y la de la tecnología, ya que se toma como indicador la investigación y desarrollo $(I+D)$ en su conjunto.

Los modelos mesoeconómicos han adaptado la función de producción para analizar la influencia de la investigación pública y privada en los resultados innovadores de diferentes territorios, como estados, regiones, provincias, áreas metropolitanas (para una revisión de estos estudios, véase Barge-Gil y Modrego, 2006). Estos modelos se empezaron a aplicar en Estados Unidos a finales de los años ochenta y principios de los años noventa (Jaffe, 1989, Acs et al., 1992; Feldman y Florida, 1994; Anselin y Varga, 1997, entre otros) y después se han extendido a varios paises europeos (Audretsch y Vivarelli, 1996; Autant-Bernard, 2001, Fisher y Varga, 2003; del Barrio-Castro y García-Quevedo, 2005). Aunque se sigue sin distinguirse la "I" de la "D", al menos se puede separar el impacto obtenido por la I+D universitaria del alcanzado por la I+D empresarial. La evidencia empírica es muy robusta y muestra que la influencia de la I+D universitaria en los resultados innovadores de los territorios es muy elevada. Esta influencia tiene lugar por una doble vía, tanto de forma directa como indirecta, a través del efecto positivo que la I+D universitaria ejerce en la I+D empresarial, con un impacto geográficamente localizado.

Los estudios microeconométricos han tratado de medir la tasa de rendimiento de la inversión privada en investigación y desarrollo (para un revisión, véase Griliches, 1995). A pesar de los diferentes problemas metodológicos planteados en estos estudios, todos ellos señalan que la tasa social de rendimiento excede a la privada y en la mayoría de los casos resulta ser más del doble.

En esta línea, las mayores aportaciones en la medición de los beneficios económicos de la investigación básica han sido realizadas por Mansfield (1991; 1998). A partir de una muestra de 76 grandes empresas de siete sectores, estimó que el $11 \%$ de los nuevos productos y el $9 \%$ de los nuevos procesos desarrollados en los últimos diez años hubieran sufrido un retraso sustancial o no se hubieran 
podido desarrollar sin la investigación académica reciente (de los 15 años previos). Mansfield (1991) estima que estos nuevos productos habían sido responsables del $3 \%$ de las ventas de las empresas en el último año del estudio mientras que los nuevos procesos habían reducidos sus costes en un $1 \%$. Además, el $8 \%$ de los nuevos productos y el $6 \%$ de los nuevos procesos habian sido desarrollados con importante ayuda de la investigación académica reciente, lo que suponía otro $2,1 \%$ de las ventas y un ahorro de costes añadido de 1,6\%. A partir de estos resultados, Mansfield (1991) estima que la tasa de rendimiento de la investigación académica era del $28 \%$. El propio autor advierte de que sus resultados son un intento más o menos afinado de estimación de dicha tasa y pone de manifiesto los múltiples problemas metodológicos encontrados. Entre ellos, cabe destacar que el período temporal de 15 años es relativamente reducido, ya que otros autores han puesto de manifiesto que el desfase temporal entre los descubrimientos científicos y su impacto económico se halla en torno a 20 ó 30 años (Adams, 1990). Como sostienen David et al. (1992), aunque los "productos tangibles" de la investigación básica (por ejemplo, nuevos productos y procesos) podrían ser un posible resultado de la investigación, generalmente las actividades complementarias que se requieren para obtener valor económico de estos resultados son enormes. Sin embargo, estos estudios no tienen en cuenta estos factores, por lo que deben realizar "supuestos heroicos", que pueden llevar a conclusiones erróneas.

\subsection{Encuestas y estudios de caso}

Los estudios que utilizan encuestas y métodos de caso ofrecen una perspectiva mucho más rica y detallada acerca de los beneficios económicos de la ciencia. Dentro de estos estudios se puede distinguir aquellos que se basan en encuestas o casos orientados a capturar el punto de vista del tejido empresarial (Arundel et al., 1995; Klevorick et al., 1995; Ham y Mowery, 1998; Cohen et al., 2002b), o a conocer el punto de vista de los científicos (Meyer-Krahmer y Schmoch, 1998; Agrawal y Henderson, 2002; Schartinger et al., 2002; D'Este y Patel, 2007).

Este tipo de trabajos muestra que la influencia de la ciencia en la economía tiene lugar a través de canales muy variados y que aquéllos a los que la literatura económica y la agenda política ha prestado más atención, como las patentes y licencias, se encuentran entre los menos im- portantes (Agrawal, 2001; Perkmann y Walsh, 2007). De hecho, las empresas consideran que las publicaciones, los contactos informales, la contratación de trabajadores y las reuniones y conferencias son las principales vías de influencia (Arundel et al., 1995; Cohen et al., 2002b). Por su parte los científicos opinan que la investigación, en colaboración o bajo contrato, la consultoría, las reuniones y conferencias y los contactos informales son los principales canales a través de los que su conocimiento fluye hacia el sector empresarial (Meyer-Krahmer y Schmoch, 1998; Agrawal y Henderson, 2002; Schartinger et al., 2002; D'Este y Patel, 2007).

Un segundo resultado de este grupo de estudios demuestra que la influencia de la ciencia en la economía varía en gran medida en función de la disciplina científica y del sector económico considerado. Klevorick et al. (1995) utilizan el concepto de "oportunidad tecnológica" para expresar las posibilidades de avance tecnológico en cada sector económico y consideran que el determinante a largo plazo más poderoso de estas oportunidades es el avance de la ciencia básica. En su estudio, obtienen que las disciplinas de ciencia aplicada y las relacionadas con la ingeniería son muy valoradas por un amplio espectro de empresas, pertenecientes a distintos sectores, pero que aquellas disciplinas más cercanas a la ciencia básica, como matemáticas, física o biología son consideradas relevantes por empresas localizados en algunos sectores concretos de alta tecnología, como pueden ser el farmacéutico o el biotecnológico. Sin embargo, las disciplinas más cercanas a la ciencia básica tienen, además, un impacto indirecto muy relevante, a través de su influencia en las disciplinas de ciencia aplicada y en la ingeniería. En general, el mapa de las relaciones entre las distintas disciplinas científicas y los distintos sectores económicos es muy complejo y no puede ser reducido a una clasificación simple entre sectores basados en ciencia y sectores no basados en ciencia (Perkmann y Walsh, 2007). Los diferentes sectores presentan grandes diferencias en el número de disciplinas relevantes, en el grado de relevancia de cada una de ellas y en los canales más importantes a través de los que tiene lugar la influencia, pero todos ellos se benefician de los avances en la actividad científica, de forma directa o indirecta y con mayores o menores retardos.

Algunos autores (Salter y Martín, 2001) han desarrollado una clasificación de los distintos tipos de beneficios económicos que se desprenden de la actividad científica, 
basándose en la complejidad y variedad de canales a través de los que la influencia tiene lugar:

1. Incremento del stock de conocimiento: los nuevos descubrimientos científicos suponen incrementos en el stock de conocimiento al que las empresas pueden recurrir cuando se encuentran con problemas que reclaman soluciones innovadoras. De hecho, la "ciencia vieja" es, en general, mucho más relevante para los procesos innovadores empresariales que los nuevos descubrimientos científicos (Kline y Rosenberg, 1986; Bender y Laestadius, 2005). Además, y esto es de gran importancia, se demuestra que los proyectos que fallan también resultan útiles, ya que suministran información acerca de dónde no buscar soluciones (David et al., 1992).

2. Formación de nuevos estudiantes: la relación entre la ciencia y la academia permite que los licenciados se incorporen al tejido empresarial no sólo con el conocimiento acerca de la investigación científica reciente, sino con la habilidad de resolver problemas complejos mediante soluciones imaginativas. Por eso, resulta vital que los sistemas educativos respondan desde los primeros niveles a la necesidad de crear en los alumnos unas bases sólidas de lo que constituye un largo proceso de aprendizaje permanente que les permita enfrentarse a los problemas reales y tener iniciativa y espíritu emprendedor (Modrego y Barge-Gil, 2004).

3. Nuevos instrumentos y metodologías: los retos afrontados por la ciencia llevan a los investigadores a diseñar nuevo equipamiento, técnicas de laboratorio y métodos analíticos adaptados a problemas específicos (Salter y Martin, 2001) que, al difundirse, consiguen un gran impacto económico, como ha sido destacado en los trabajos de los historiadores (véase, por ejemplo, Rosenberg, 1992).

4. Formación de redes e interacciones sociales: las empresas consideran que los contactos informales, que se articulan principalmente a través de redes, constituyen un importante vehículo de comunicación con la ciencia. A través de las redes se accede a una variedad mucho mayor de fuentes de información que a través del mercado (Callon, 1994). Además, la ciencia se caracteriza por sus vínculos internacionales, por lo que pueden servir de puente para que el tejido productivo acceda a conocimiento generado en otros paises (van Helleputte y Reid, 2004).
5. Solución de problemas tecnológicos concretos: Aunque generalmente se asocia la influencia de la ciencia exclusivamente con las innovaciones denominadas radicales, en muchas ocasiones la ciencia ofrece forma de resolver problemas (Cohen et al., 2002b). Las actividades menos apreciadas, como la consultoría y la investigación bajo contrato son muy utilizadas y consideradas muy relevantes tanto por la industria como por los científicos (Meyer-Krahmer y Schmoch, 1998; Cohen et al., 2002b).

6. Creación de nuevas empresas. Salter y Martin (2001) expresan sus dudas acerca de la importancia real de esta vía de influencia en la economía. Sin embargo, en los últimos años ha crecido espectacularmente el número de spin-offs de base científica, así como la atención que los economistas y los políticos dedican a este fenómeno. A pesar de ello, todavía no existe una evidencia empírica mínimamente consolidada acerca del grado de éxito de estas iniciativas ni de los factores que pueden contribuir a su supervivencia y desarrollo.

\section{El debate ACtUAL: CIENCIA ABIERTA VS DERECHOS DE PROPIEDAD INTELECTUAL}

La ciencia abierta, entendida como el libre acceso al conocimiento científico, tiene su origen a finales del siglo XVI y principios del siglo XVII, desbancando al régimen de secretismo predominante en la búsqueda de los "secretos de la naturaleza". Este cambio es un aspecto distintivo y vital de la revolución científica, que cristalizó en una serie de nuevas convenciones, sistemas de incentivos y mecanismos institucionales que reforzaron el compromiso de los investigadores hacia la rápida revelación y diseminación de los nuevos descubrimientos. Este sistema constituyó una respuesta funcional a los problemas de información asimétrica existentes en el sistema de mecenazgo, como consecuencia del creciente peso de las matemáticas en el lenguaje científico (David, 2004). En los siglos XVII y XVIII se institucionalizó el régimen de ciencia abierta bajo el patrocinio de academias estatales, como la Royal Society of London o la Académie Royale des Sciences.

Los principales beneficios del régimen de ciencia abierta son la rápida crítica y validación de los descubrimientos, que asegura la calidad de los mismos, la reducción de un exceso de duplicación de los esfuerzos de investigación, el 
aumento del espacio para buscar complementariedades y para desarrollos acumulativos de la ciencia y los desbordamientos beneficiosos entre programas de investigación (Cohen et al., 2002a; David, 2004).

En estos momentos, algunos autores muestran su preocupación por la posibilidad de que se esté produciendo un cambio de régimen, hacia un modelo de ciencia cerrada y basada en la propiedad privada. Se suele considerar que el hito principal de este cambio de régimen ha sido la BayhDole Act, aprobada en el Congreso de los Estados Unidos en 1980. Dicha ley tenía como objetivo facilitar las patentes y licencias universitarias de las investigaciones llevadas a cabo con financiación pública, como una medida para fomentar la transferencia tecnológica entre las universidades y el tejido empresarial.

Existe gran controversia acerca de los efectos de la BayhDole Act (Sampat, 2006). Por una parte ha sido considerada como un éxito absoluto e, incluso, como una de las claves del incremento de productividad alcanzado por Estados Unidos a finales de los años noventa (OCDE, 2000). Por otra parte, algunos autores (Mowery y Sampat, 2005) defienden que su principal efecto fue acelerar la tendencia en las universidades a patentar los resultados de la investigación, que había comenzado años atrás. Por último, otros autores (Nelson, 2006) argumentan que sus efectos negativos han superado a los positivos y reclaman una reformulación de la ley.

Sampat (2006) señala que los análisis más optimistas del efecto de la Bayh-Dole Act no tienen en cuenta qué hubiera ocurrido si ésta no se hubiese instaurado, en un doble sentido. Por una parte, la tendencia creciente de las patentes universitarias ya había comenzado. Por otra, en muchas ocasiones la transferencia de conocimiento hu-

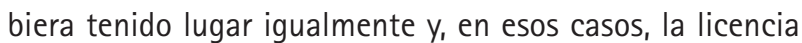
de la propiedad intelectual funciona como un impuesto para las empresas que hacen uso de los resultados de la investigación. En esta misma línea señala la importancia de considerar la influencia que la Bayh-Dole Act ha tenido sobre los diferentes canales de transferencia de conocimiento, desde la universidad hasta el sector productivo que, como se ha visto, son considerados por las empresas más importantes que las patentes y licencias.

Los defensores del modelo de la ciencia abierta argumen$\tan ($ Nelson, 2004; 2006) que el conocimiento científico no es un producto final, sino que su uso principal es ser un recurso sobre el que se pueda desarrollar la investigación futura. Desde este punto de vista, patentar los resultados de la investigación básica, supone en gran medida un bloqueo de la investigación futura $y$, dado que la investigación se halla sujeta a incertidumbre, resulta socialmente deseable que se exploren varios caminos simultáneamente y exista competencia entre diversos agentes que buscan aplicar la investigación. Mientras los derechos de propiedad industrial generalmente se han utilizado para conceder monopolios en el mercado de productos finales, la extensión a la ciencia básica concede monopolios sobre líneas de investigación.

Desde el punto de vista evolucionista, si se tiene en cuenta la incertidumbre inherente en el proceso de aplicación de la ciencia y desarrollo de los productos, junto con el carácter acumulativo del desarrollo del conocimiento, restringir la competencia en este proceso puede transformarse en un daño a la sociedad en el medio y largo plazo. Además, como se ha visto anteriormente, las patentes y licencias no son consideradas ni por empresas ni por científicos como uno de los canales más importantes de transferencia de conocimiento. Por el contrario, son los canales relacionados con la ciencia abierta (publicaciones, conferencias, contactos informales) y las relaciones basadas en la prestación de servicios de investigación y consultoría.

\section{Conclusiones}

La importancia que el conocimiento científico tiene en el progreso económico y social de la llamada sociedad del conocimiento hace cada vez más atractivo profundizar y actualizar el estado del arte en la relación entre ciencia y economía. Aunque en términos generales se puede afirmar que una sociedad donde la cultura científica forma parte de su acerbo cultural tiene una ventaja comparativa innegable en relación con otras en las que la ciencia todavía no ha conseguido alcanzar esos niveles de aceptación y valoración, no se dispone de un conocimiento contrastado y concluyente sobre los múltiples factores que intervienen en dicha relación.

A lo largo de este trabajo, se ha pretendido hacer una presentación de las principales aproximaciones al estudio 
de la relación entre ciencia y economía, una relación que no es unilateral y que está condicionada por el comportamiento y las interacciones de múltiples agentes individuales e institucionales.

La principal conclusión, y la más obvia, es que ciencia y economía están intimamente relacionadas y que el conocimiento científico forma parte de la riqueza de las naciones.

La segunda conclusión, y quizás la más atractiva, es que hay mucho camino por recorrer para llegar a entender una relación de tal complejidad, donde hay que tener presente de forma permanente el carácter dinámico tanto de la ciencia como de la economía, y su relación con la sociedad. El tratamiento que desde la economía se ha dado al análisis de la ciencia presenta serias deficiencias; el "resurgir" del enfoque evolucionista puede contribuir a subsanar algunas de ellas. Esto tiene repercusiones no sólo desde el punto de vista del análisis de dicha relación, sino de las actuaciones orientadas a potenciarla en beneficio de los ciudadanos. Actuaciones que han de evolucionar de acuerdo a los resultados que se pretendan alcanzar en cada momento y que conlleva diseñar un seguimiento continuo de la eficacia y de la eficiencia de cada una de ellas.
La tercera conclusión es que la complejidad de la relación entre ciencia y economía dificulta la estimación del impacto que el progreso científico puede tener en el progreso económico y social. Los distintos tipos de impacto, los desfases temporales entre la obtención de un resultado y su puesta en valor, la variedad de canales de difusión y explotación del conocimiento generado, los múltiples agentes e instituciones que intervienen en el proceso, las características diferenciales de disciplinas científicas y sectores productivos, reclaman un esfuerzo metodológico, de recogida de información y de análisis que facilite la identificación a distintos niveles los diferentes tipos de impacto.

La cuarta y última conclusión es una llamada de atención que, en cierta medida, resume las conclusiones anteriores. El debate clave para el futuro sobre ciencia abierta y derechos de propiedad es un ejemplo de la precaución que hay que tomar a la hora de adoptar medidas. Aun a riesgo de resultar reiterativos, es preciso resaltar que el carácter dinámico de la actividad científica, de la actividad económica y de la sociedad, requiere planteamientos flexibles que se adecuen a las circunstancias, a las necesidades y a los problemas que se pretenden resolver y una valoración permanente de los resultados obtenidos.

\section{NOTAS}

1 Este concepto ha sido principalmente aplicado al ámbito empresarial o territorial, al entender a éstos como "receptores del conocimiento". En nuestra opinión, el concepto debería ser extendido para tratar las capacidades otras organizaciones, como los grupos de investigación que les permitan obtener el mayor provecho del conocimiento que reside en el mundo empresarial.

2 Esto no supone excluir la utilización de técnicas estadisticas y econométricas al análisis de la información generada por las encuestas.

\section{BIBLIOGRAFÍA}

Acs, Z.; Audretsch, D. y Feldman, M. (1992): "Real effects of academic research: comment", The American Economic Review, 82(1), 363-367.

Agrawal, A. (2001): "University -to-industry knokwledge transfer: literature review and unanswered questions", International Journal of Management Reviews, 3(4), 285-302.

Agrawal, A. y Henderson, R. (2002): "Putting patents in context: Exploring knowledge transfer from MIT", $M a-$ nagement Science, 48(1), 44-60.

Anselin, L. y Varga, A. (1997): "Local geographic spillovers between university
Aceptado: 22 de julio de 2008

ARBOR CLXXXV 738 julio-agosto [2009] 757-766 ISSN: 0210-1963 
research and high technology innovations", Journal of Urban Economics, 42, 422-448.

Arrow, K. (1962): "Economic welfare and the allocation of resources for invention", en Nelson, R. (ed.), The rate and direction of inventive activity, Princeton University Press, Princeton, pp. 609-625.

Arundel, A.; Van de Paal, G. y Soete, L. (1995): PACE Report: Innovation Strategies of Europe's Largest Firms: Results of the PACE Survey for Information Sources, Public Research, Protection of Innovations, and Government Programmes, Final Report, MERIT, University of Limburg, Maastricht.

Audretsch, D. y Vivarelli, M. (1996): "Firms size and R\&D spillovers: Evidence from Italy", Small Business Economics, 8(3), 249-258.

Autant-Bernard, C. (2001): "Science and knowledge flows: evidence from the French case", Research Policy, 30, 1069-1078.

Barge-Gil, A. y Modrego, A. (2006): "External Sources of Knowledge: What are the functions they perform to foster innovative environments?", en Remenyi, D. (ed.), "ECEl 2006", European Conference on Entrepreneurship and Innovation, 1-10, Ed. Academic Conferences Limited.

Bender, G. y Laestadius, S. (2005): "Nonscience based innovativeness-on capabilities relevant to generate profitable novelty", en Bender, G.; Jacobson, D. y Robertson, P. L. (eds.), "Non-ResearchIntensive Industries in the Knowledge Economy", Journal for Perspectives on Economic Political and Social Integration, Special Edition, Volume XI/2005, No. 1-2, Katholische Universität S. y Lublin/PL, pp. 123-170.

Bush, V. (1945): Science, the endless frontier, Washington DC, US GPO.

Cohen, W. y Levinthal, D. (1989): "Innovation and learning: the two faces of
R\&D", The Economic Journal, 99 (397), 569-596.

Cohen, W. y Levinthal, D. (1990): "Absorptive capacity: a new perspective on learning and innovation", Administrative Science Quarterly, 35 (1), 128-152.

Cohen, W. M.; Florida, R.; Randazzese, L. y Walsh, J. (2002a): "Industry and the Academy: Uneasy partners in the cause of technology advance", en Noll, R. (ed.): Challenge to the Research University, Washington, DC, Brookings Institution.

Cohen, W. M.; Nelson, R. y Walsh, J. (2002b): "Links and impacts: the influence of public research on industrial R\&D", Management Science, 48(1), 1-23.

D'Este, P. y Patel, P. (2007): "Universityindustry linkages in the UK: What are the factors underlying the variety of interactions with industry?", Research Policy, 36, 1295-1313.

Dasgupta, P. y David, P. (1994): "Toward a new economics of science", Research Policy, 23(5), pp. 487-521.

David, P. (2004): "Understanding the emergence of 'open science' institutions: functionalist economics in historical context", Industrial and Corporate Change, 13(4), 571-589.

David, P.; Mowery, D. y Steinmuller, W. (1992): "Analysing the economic payoffs from basic research", Economics, Innovation and New Technology, 2, 73-90.

Del Barrio-Castro, T. y Garcia-Quevedo, J. (2005): "Effects of university research on the geography of innovation", Regional Studies, 39(9), 1217-1229.

Dosi, G. (1988): "The nature of innovative process", en Dosi, G.; Freeman, C.; Nelson, R.; Silverberg, G. y Soete, L. (eds.), Technical Change and Economic Theory, London, Frances Printer y New York, Columbia University Press, pp. 221-238.
Dosi, G. y Nelson, R. (1998): "An introduction to Evolutionary Theories in Economics", Journal of Evolutionary Economics, 4(3), 153-172.

Feldman, M. y Florida (1994): "The geographic sources of innovation: technological infrastructure and product innovation in the United States", Annals of the Association of American Geographers, 84(2), 210-229.

Fischer, M. y Varga, A. (2003): "Spatial knowledge spillovers and university research: Evidence from Austria", The Annals of Regional Science, 37, 303-322.

Gibbons, M.; Limoges, H.; Nowotny, S.; Schwartzman, S.; Scott, P. y Trow, M. (1994): The new production of knowledge, London, Sage.

Griliches, Z. (1995): "R\&D and productivity", en Stoneman, P. (ed.), Handbook of Industrial Innovation, Blackwell Publishers, Londres, 52-89.

Grossman, G. y Helpman, E. (1989): "Comparative advantage and long-run growth", American Economic Review 89, 796-815.

Ham, R. M. y Mowery, D. (1998): "Improving the effectiveness of public-private R\&D collaborations: case studies at a US weapons laboratory", Research Policy, 26, 661-675.

Jaffe, A. (1989): "Real effects of academic research", The American Economic Review, 79(5), 957-970.

Klevorick, A.; Levin, R.; Nelson, R. y Winter, S. (1995): "On the sources and significance of inter-industry differences in technological opportunities", Research Policy, 24, 185-205.

Kline, S. y Rosenberg, N. (1986): "An overview of innovation", en Landau, R. y Rosenberg, N. (eds.), The Positive Sum Strategy: Harnessing Technology for Economic Growth, National Academic Press, Washington DC, pp. 273-305.

Lane, P.; Koka, B. y Pathak, S. (2006): "The reification of absorptive capacity: a 
critical review and rejuvenation of the construct", Academy of Management Review, 31(4), 833-863.

Leydesdorff, L. y Etzkowitz, H. (1996): "Emergence of a triple helix of university-industry-government relations", Science \& Public Policy, 23, 279-286.

Mansfield, E. (1991): "Academic research and industrial innovation", Research Policy, 20, 1-12.

Mansfield, E. (1998): "Academic research and industrial innovation: an update of empirical findings", Research Policy, 26, 773-776.

Marshall (1890): Principles of Economics, London, Macmillan $(1890,1927), 8^{\text {th }}$ Edn.

Martin, B. (2003): "The changing social contract for science and the evolution of the university", en Geuna, A.; Salter, A. y Steinmuller, W. (eds.), Science and innovation. Rethinking the rationales for funding and governance, Edward Elgar, Cheltenham, UK, Northampton, MA, USA, 7-29.

Metcalfe, S. (1995): " The Economic Foundations of Technology Policy: Equilibrium and Evolutionary Persepectives", en Handbook of the Economics of Innovation and Technological Change, pp. 409-512.

Metcalfe, S. (1997): "Technology systems and technology policy in an evolutionary framework", en Archibugi, D. y Michie, J., Technology globalisation and economic performance, Cambridge University Press, pp. 268-296.

Meyer-Krahmer, F. y Schmoch, U. (1998): "Science-based technologies: university-industry interactions in four fields", Research Policy, 27, 835-851.

Modrego, A. y Barge-Gil, A. (2004): "Innovación y formación: bases para un desarrollo sostenido", Ekonomiaz, 56(2), 178-207.
Mowery, D. y Sampat, B. N. (2005): "The Bayh-Dole Act of 1980 and university-industry technology transfer. A model for other OECD governments?", Journal of Technology Transfer, 30 (1/2), 115-127.

Nelson, R. y Soete, L. (1988): "Policy conclusions", en Dosi et al. (eds.) (1988), pp. 631-635.

Nelson, R. R. y Winter, S. G. (1982): An Evolutionary Theory of Economic Change, Belknap Press, Cambridge, Mass. y London.

Nelson, R. R. (1959): "The simple economics of basic scientific research", The Journal of Political Economy, 67(3), pp. 297-306.

Nelson, R. R. (2004): "The market economy, and the scientific commons", Research Policy, 33, 455-471.

Nelson, R. R. (2006): "Reflections on the simple economics of basic scientific research", Industrial and Corporate Change, 15(6), 903-917.

OCDE (2000): A new economy?, Paris, OCDE. Tomado de Mowery y Sampat (2005).

OTA (1986): "Research funding as an investment: can we measure the returns?", A technical memorandum, Office of Technology Assessment, US Government Printing Office, Washington DC. Tomado de Salter y Martin (2001).

Perkmann, M. y Walsh, K. (2007): "University-industry relationships and open innovation: Towards a research agenda", International Journal of Management Reviews, 9(4), 259-280.

Polanyi, M. (1962): "The republic of science", Minerva, 1(1), pp. 54-73.

Romer, P. (1990): "Endogenous technological change", Journal of Political Economy, 98, 71-102.

Rosenberg, N. (1992): "Scientific instrumentation and university research", Research Policy, 19, 165-174.
Rosenberg, N. y Nelson, R. (1994): "American universities and technical advance in industry", Research Policy, 23, 323-348.

Salter, A. y Martin, B. (2001): "The economic benefit of publicly funded basic research: a critical review", Research Policy, 30, pp. 509-532.

Sampat, B. N. (2006): "Patenting and US academic research in the $20^{\text {th }}$ century: The world before and after Bayh-Dole", Research Policy, 35, 772-789.

Schartinger, D.; Rammer, C.; Fischer, M. y Fröhlich, J. (2002): "Knowledge interactions between universities and industry in Austria: sectoral patterns and determinants", Research Policy, 31, 303-328.

Schumpeter, J. (1943): Capitalism, Socialism, and Democracy, London George Allen and Unwin (5 ${ }^{\text {th }}$ Edition 1976).

Schumpeter, J.: The Theory of Economic Development, Cambridge, MA, Harvard University Press (1934, original edition 1912).

Solow, R. (1957): "Technical change and the aggregate production function", Review of Economics and Statistics, 39, 214-231.

Stephan, P. (1996): "The economics of science", Journal of Economic Literature, 34(3), pp. 1199-1235.

Van Helleputte, J. y Reid, A. (2004): "Tackling the paradox: can attaining global research excellence can be compatible with local technology development?", $R \& D$ Management, 34(1), 33-44.

Veblen, T. (1898): "Why is economics not an evolutionary science?", Quarterly Journal of Economics, 12, 374-397.

Verspagen, B. (1992): "Endogenous innovation in neoclassical growth models: a survey", Journal of Macroeconomics, 14, 631-662. 